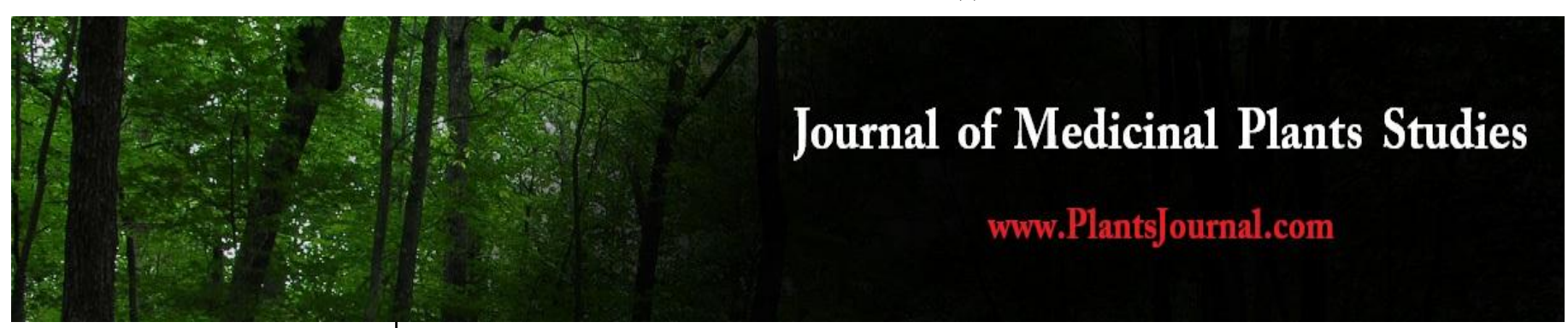

ISSN (E): 2320-3862 ISSN (P): 2394-0530 www.plantsjournal.com JMPS 2022; 10(1): 87-97 (C) 2022 JMPS

Received: 06-11-2021

Accepted: 09-12-2021

Prameela Rapaka Department of Botany, M.R. Degree College, Vizianagaram, Andhra Pradesh, India

Corresponding Author: Prameela Rapaka Department of Botany, M.R. Degree College, Vizianagaram, Andhra Pradesh, India

\section{Fort flora of vizianagaram}

\section{Prameela Rapaka}

DOI: https://doi.org/10.22271/plants.2022.v10.i1b.1366

\section{Abstract}

Vizianagaram Fort is situated at the heart of the town with illuminates many lives and also home for both Flora and Fauna. The present paper explores the Flora of Vizianagaram fort. It is not only Royal palace or educational institution or tourist place but also a home of biodiversity, having good number of Flora and Fauna. The fort plants categorized into Mushrooms, Pteridophytes, Gymnosperm, Herbs (decumbent herbs, tuberous herbs), Shrubs, Trees ( Shade trees, fruit yielding, Palms, Ornamental tree), Creepers, Hydrophytes, and Xerophytes etc. of 279 species belongs to 71 families. Economically fort plants are categorised into Medicinal plants, Ornamental plants, Weeds, Fruit yielding plants, Vegetable plants, Fibre yielding plants, Wood yielding plants, Fodder plants and Dye yielding plants.

Keywords: Flora, Fort, Sri Dr. P.V.G. Raju, Vizianagaram

\section{Introduction}

Vizianagaram fort was built in the year 1713 as a royal palace by Vijaya Rama Raju, also known as Anand Raju-I, the then Maharaja of Vizianagaram. It is situated in Vizianagaram which is away from 50 kilometres to the City of Viakhapatnam and 18 kilometres to the Bay of Bengal. During the year 1958 Rajah Saheb of Vizianagaram Dr. P.V.G.Raju donated his palace for educational institutions. "The most truly generous persons are those who give silently without hope of praise or reward" (Carol Ryrie Brink). "True generosity is an offering; given freely and out of pure love. No strings attached. No expectations" (Suze Orman). "Generosity is the most natural outward expression of an inner attitude of compassion and loving- kindness" (Dalai Lama). These quotes are not enough to describe Rajah Saheb Dr.P.V.G. Raju. In 1946 he decided to give-up the Zamindari (Estate) without taking any compensation. He created a trust in the name of his beloved father "Maharajah Alaknarayana Society of Arts and Science MANSAS" on $12^{\text {th }}$ November 1958 . He created permanent income to the Trust by gifting all his properties, which includes 6,622 acres of dry land and 2,350 acres of wetland. Now more than a dozen institutions from primary classes to post graduate college are under the management of MANSAS. Another remarkable event which gives pride not only to Dr.P.V.G.Raju but also to the state of Andhra Pradesh and to the nation as a whole was that he donated 100 acre of the land to the central Government for locating Sainik School at Korukonda village, near Vizianagaram town. Fort, Dr.P.V.G.Raju statue images given in Fig 1.

It is not only Royal palace or educational institution or tourist place but also a home of biodiversity, having good number of Flora and Fauna. Here we can see plenty of diversity in Flora and Fauna. Birds (Parrots, Pigeons, Crows, Common Myna, Cattle Egret), bats, butterflies and their babies, Beatles and Bugs, Bees, snakes, garden lizards, Squirrels millipedes and Centipedes are common fauna in the fort. There are Killers (White lies, Beatles), friends (Pollinators), Frienemies (Butterflies are helped in pollination where as their babies are Licked the plants). Fauna showed in Fig 2. The present paper focus mainly on Flora, some important plants showed in Fig 3 \& 4.

Fort flora is asset to many students and researchers. Every year all the educational institutes have been conducting exhibitions for the knowledge of students and public in Vizianagar Utsav on the occasion of Pyditalli Festival. Part of this Department of Botany, M.R.College for Women has been exhibiting some posters and live plants growing in the campus. Medicinal plants, Poisonous plants, Ornamental plants, Aromatic plants, Hydrophytes and Tuberous plants and creating awareness among the people about the plants and also conservation status. Some of the rare and endangered species are conserving in the fort campus, because here there are no anthropological activities and no animal threat. 


\section{Materials and methods \\ Study area}

The fort, built of stone, is in the shape of a square of side 240 metres and rises to a height of 10 metres. The width of the wall at the top varies from 8 to 16 metres. There are two gates of entry into the fort. A moat surrounded the fort. Apart from the two main gates, there are two temples and monuments located within the fort. Two important temples are the Hanuman temple, and the lakshmi temple known as the "Kota Shakti", which is the guardian deity of the fort. It is a home of biodiversity, having good number of Flora and Fauna. A total of 279 plants are growing in the campus. Coordinates of the fort: $18^{\circ} 06^{\prime} 39^{\prime \prime} N 83^{\circ} 24^{\prime} 38^{\prime \prime} E$.

As the study area is author's work place, continuous observations were conducted since 2010. During these years some changes in the flora were noticed. Especially in annual herbs, species richness is variant and changed year to year. Few important trees were lost due to road and building construction and also Hudhud cyclone (2014) damaged some of the trees. The lost trees were Adenanthera pavonina, Parkia, Limonia acidissima, Bauhinia purpurea, Bixa orellana, Callistemon linearis, Casuarina equietifolia, Grevillea robusta and Sapindus. During the years 2019 2021 for documentation and explore the fort flora author has collected and deposited in the College herbarium after identification. Plants were identified with the help of Local floras (Flora of Vizianagaram, Flora of Srikakulam, Flora of Andhra Pradesh and also taking help from BSI, Hyderabad. Plant list given in alphabetical order, in Table 1.

\section{Results and discussion}

A total of 279 species are listed in the Table 1. There is much more diversity in the fort flora. The present paper categorize the plants into Mushrooms, Pteridophytes, Gymnosperm, Herbs (decumbent herbs, tuberous herbs), shrubs, Trees (Shade trees, fruit yielding, Palms, Ornamental tree),
Creepers, Hydrophytes, and Xerophytes etc. of 280 species belongs to 71 families. Families list given in the Table 2 .

With 24 species Fabaceae stood in first, followed by Euphorbiaceae, Apocyanaceae, Poaceae, Asparagaceae, Asteraceae, Zingiberaceae, Amaryllidaceae, Araceae, and Arecaceae. Eight families consist 3 species, thirteen families consist 2 species, seven families consist 5 species and 28 families are consist only single species. Table 3 showing 10 dominant families.

Herbs are common in the study area and occupy in the first place with 48.5 percent, next followed by trees, Shrubs, climbers and Hydrophytes. Details are shown in Table 4 with Pie chart.

Oldest trees in the study area are Artocarpus heterophyllus, Azadirachta indica, Bombax ceiba, Brownea coccinea, Kleinhovia hospital, Pithecellobium dulce, Swietenia mahagoni, Tamarindus indica and Terminalia arjuna.

Hydrophytic plants are Hydrilla verticillata, Ipomoea aquatic, Ludwigia adscendens, Najas indica, Nymphaea nouchali, Pistia stratiotes, and Spirodela polyrhiza. Fig: 5

India has 173 Invasive plant species, in the state of Andhra Pradesh about 78 Invasive plant species are growing. Some of Invasive plant species in the study area are Antigonon leptopus, Blumea lacera, Calotropis, Chloris barbata, Chromolaena odorata, Cleome rutidosperma, Cleome viscose, Corchorus aestuans, Cryptostegia grandiflora, Digera muricata, Euphorbia hirta, Euphorbia cyathophora, Evolulus nummularius, Lantana camara, Parthenium hysterophorus, Pistia stratiotes, Ravenia spectabilis, Senna alata, Spathodea campanulata, Tribulus terestris.

Economically fort plants are categorised into Medicinal plants, Ornamental plants, Weeds, Fruit yielding plants, Vegetable plants, Fibre yielding plants, Wood yielding plants, Fodder plants, Dye yielding plants and miscellaneous plants. List is given in the Table 5 with pie chart.

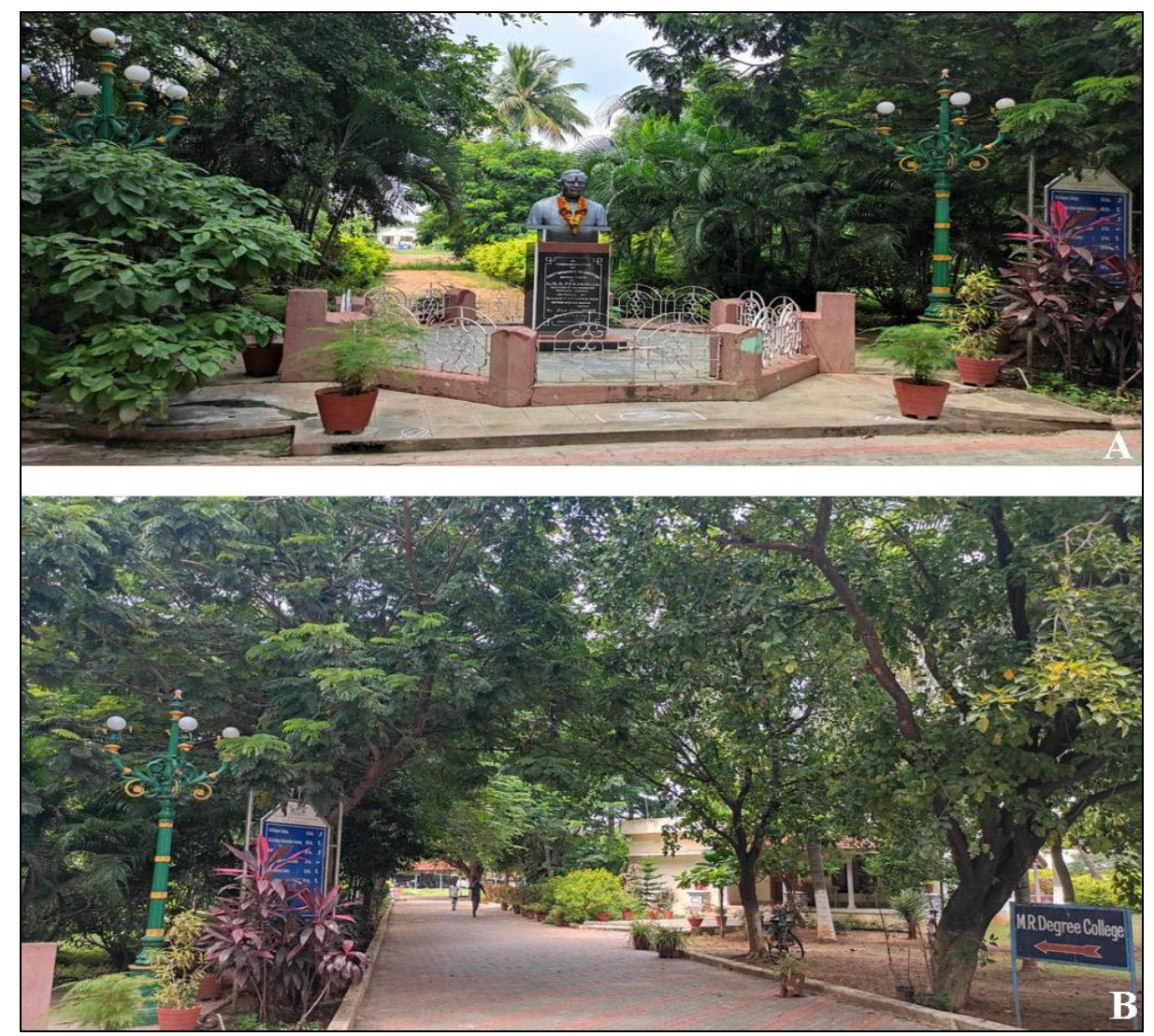



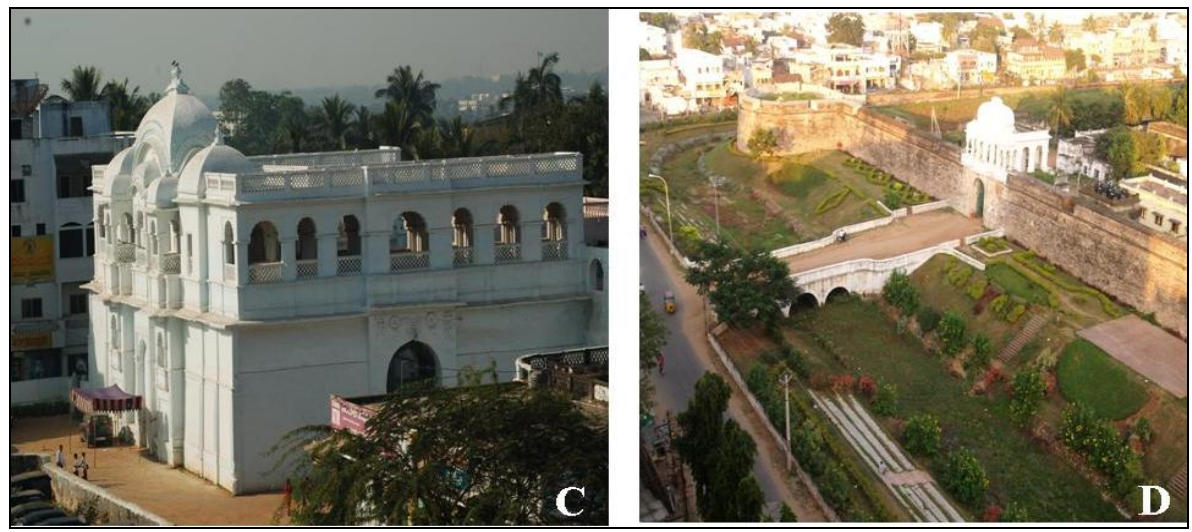

Fig 1: Fort Campus
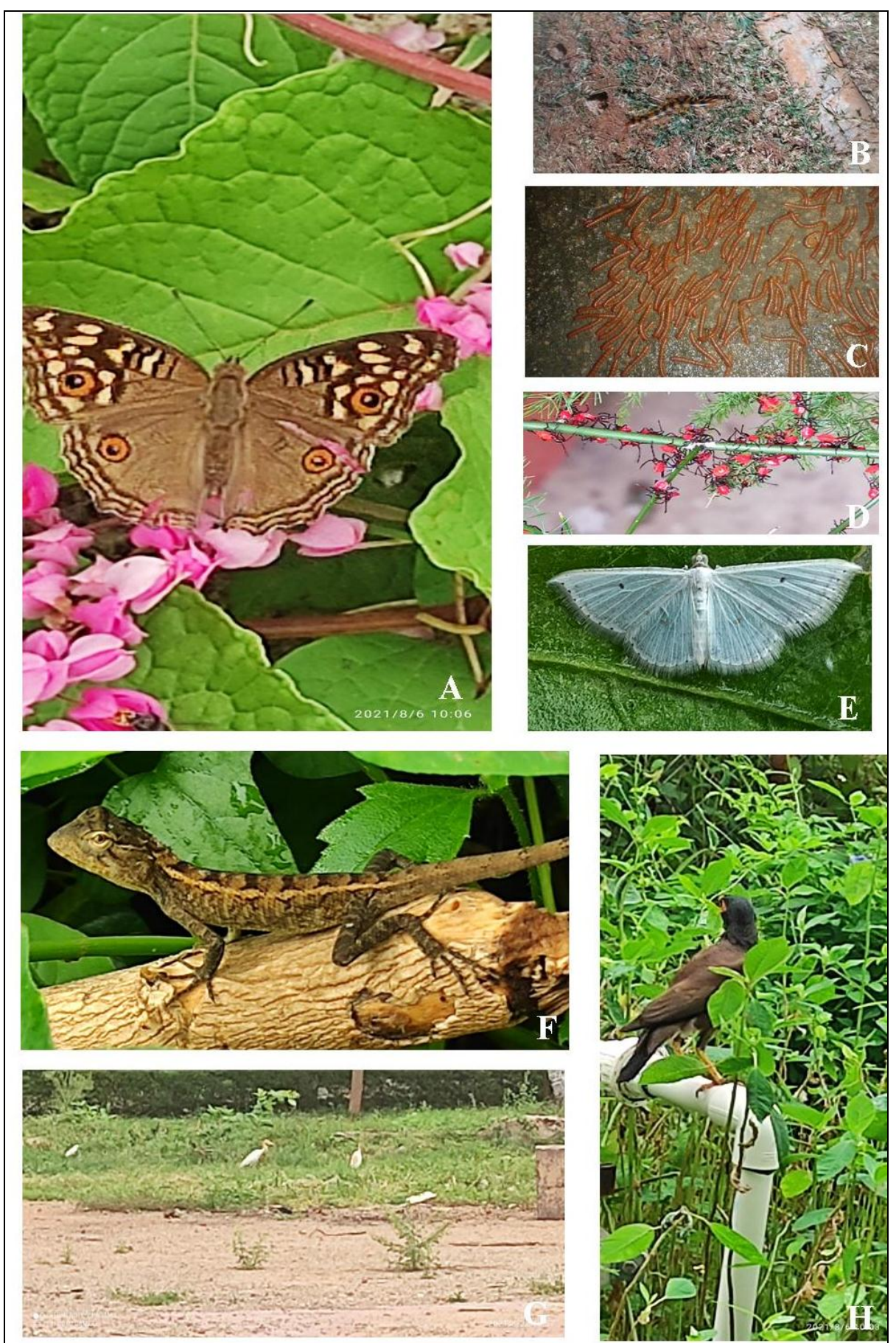

A) Butterfly； B) Centipede; C) Millipede; D) Bugs; E) Moth; F) Garden lizard; G) Common regret; H) Myna

Fig 2: Fauna 


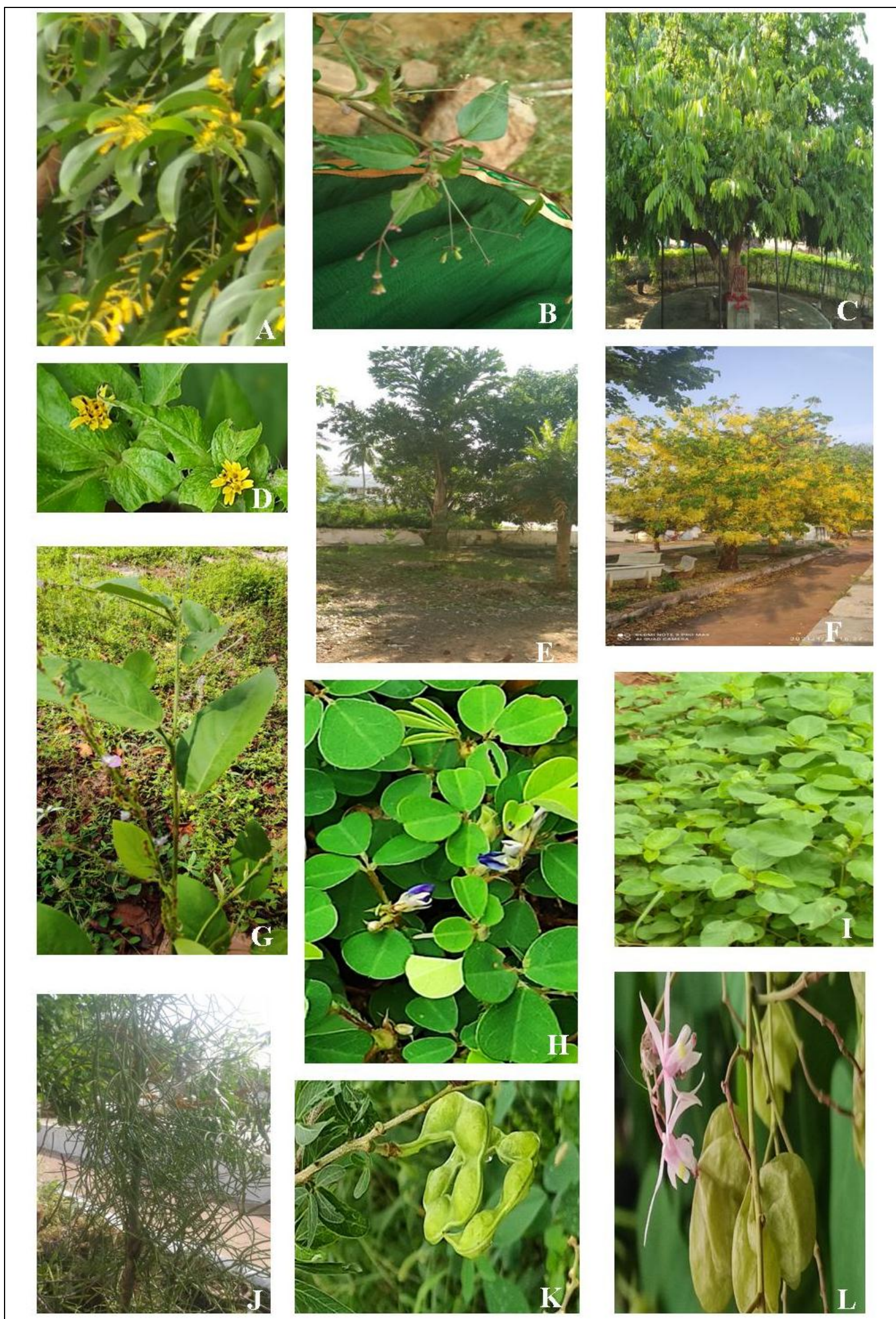

A) Acacia auriculiformis; B) Boerhaavia erecta; C) Brownea coccinia; D) Calyptocarpus vialis; E) Caryota urens; F) Cassia fistula; G) Desmodium gangeticum; H) Desmodium triflorum; I) Digera arvensis; J) Euphorbia tirucalli; K) Pithecellovium dulce; L) Kleinhovia hospita

Fig 3: Flora 

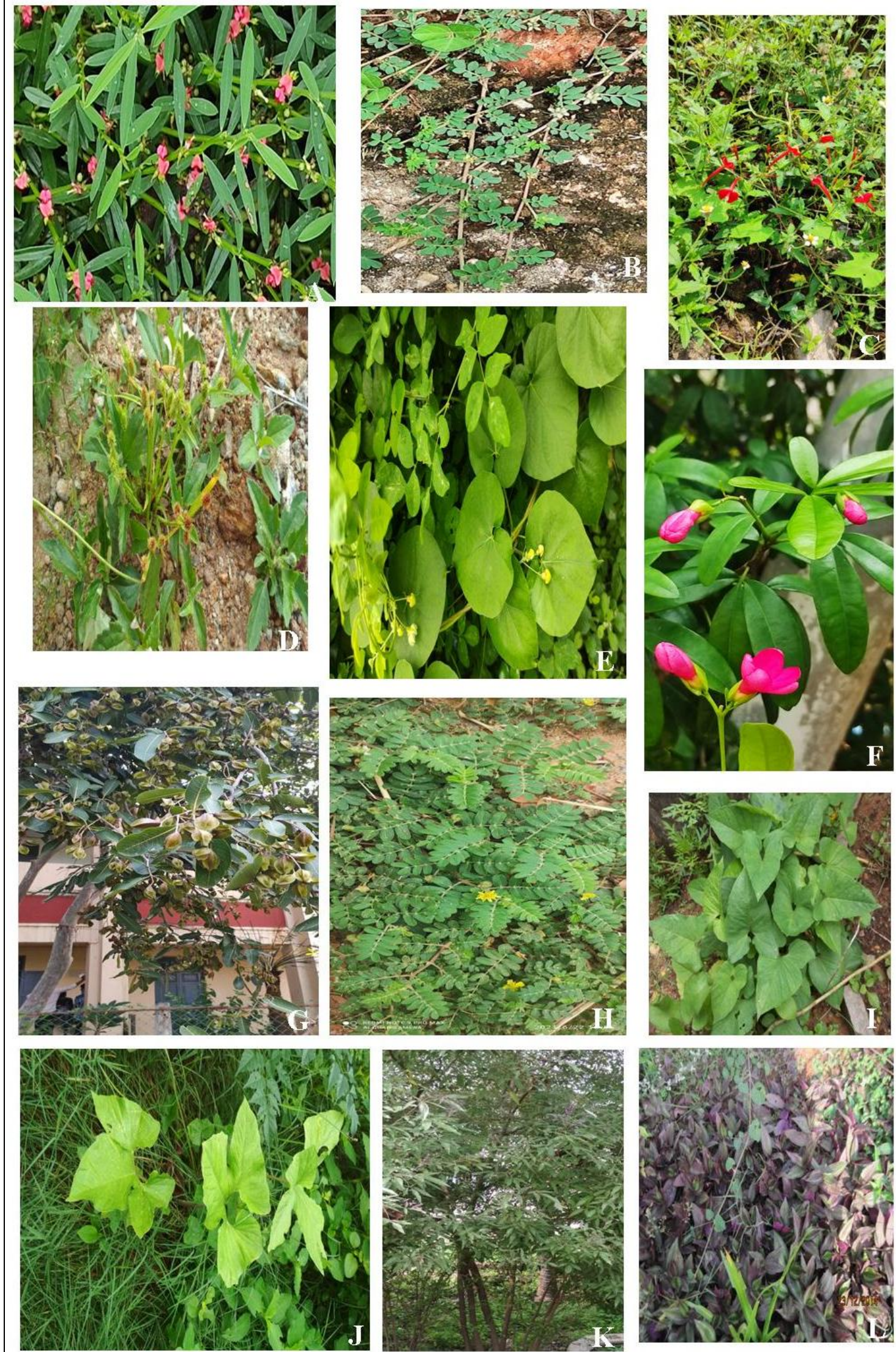

A) Indigofera linifolia; B) Indigofera linnae; C) Ipomoea hederifolia; D) Mariscus; E) Pergularia daemia; F) Ravinia spectabilis; G) Terminalia arjuna; H) Tribulus terrestris; I) Typhonium inopinatum; J) Typhonium trilobatum; K) Vitex negundo; L) Zebrena

Fig 4: Flora 

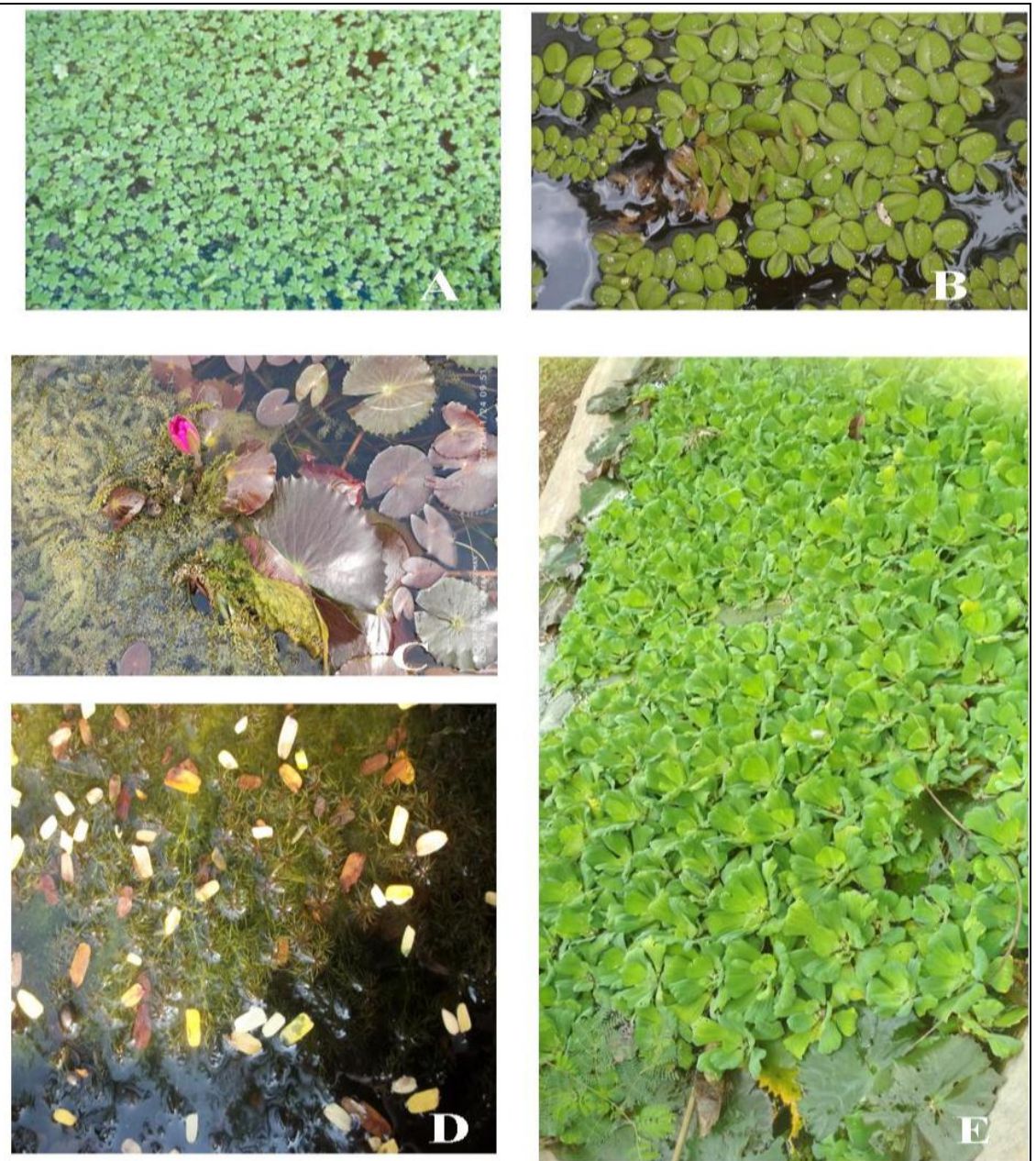

A). Azolla; B) Salvinia; C) Nymphaea; D) Najas; E) Pistia

Fig 5: Aquatic Plants

Table 1. List of the plant diversity in Fort Campus

\begin{tabular}{|c|c|c|c|c|c|}
\hline S. No & Scientific Name & Family & Common Name & Habit & Uses \\
\hline 1 & Acacia auriculiformis A.Cunn.Ex Benth & Fabaceae & Auri, earpod wattle & Tree & Ornamental and Wood yielding plant \\
\hline 2 & Acalypha hispida $\mathrm{L}$. & Euphorbiaceae & The Chenille plant & Shrub & Ornamental plant \\
\hline 3 & Acalypha indica $\mathrm{L}$. & Euphorbiaceae & Muripenda, Indian Mercury & Herb & Medicinal plant \\
\hline 4 & Acalypha wilkesiana (Jacq.) R.K.Jansen & Euphorbiaceae & Copper leaf, Jacob's coat & Shrub & Ornamental plant \\
\hline 5 & Achyranthus aspera (Forssk) Roem. \& Schult. & Amaranthaceae & Uttareni & Herb & Medicinal plant \\
\hline 6 & Acmella radicans (Jacq.) R.K.Jansen & Asteraceae & & Herb & Weed \\
\hline 7 & Adenium obesum (Forssk) Roem. \& Schult. & Apocyanaceae & & Succulent & Ornamental plant \\
\hline 8 & Adiantum aleuticum (Rupr.) C.A.Paris & Pteridaceae & Maidenhair fern & Herb & Ornamental weed \\
\hline 9 & Aegle marmelos (L.) Correa & Rutaceae & Maredu & Tree & Medicinal plant \\
\hline 10 & Aerva lanata (L.) Juss. Ex Schult & Amaranthaceae & & Herb & Medicinal plant \\
\hline 11 & Agave americana $\mathrm{L}$. & Asparagaceae & Kittanaara & Shrub & Fiber yielding \\
\hline 12 & Allamanda cathartica $\mathrm{L}$. & Apocyanaceae & Golden trumpet & Shrub & Ornamental plant \\
\hline 13 & Allamanda violacea Gardner & Apocyanaceae & & Creeper & Ornamental plant \\
\hline 14 & Alocasia macrorrhizos (L.) G.Don & Araceae & Giant Taro & Tuberous herb & Ornamental plant \\
\hline 15 & Alocasia sanderiana (Schott) G.Don & Araceae & kris plant & Tuberous herb & Ornamental plant \\
\hline 16 & Aloe vera (L.) Burm.f. & Asphodelaceae & Kalabanda & Succulent & Medicinal plant \\
\hline 17 & Alpinia calcarata (Andrews) Roscoe & Zingiberaceae & & Tuberous herb & Ornamental plant \\
\hline 18 & Alpinia speciosa (Blume) D.Dietr & Zingiberaceae & Shell Ginger & Tuberous shrub & Ornamental plant \\
\hline 19 & Alstonia scholaris (L.) R.Br. & Apocyanaceae & Edaakula chettu/ Devils tree & Tree & Wood yielding \\
\hline 20 & Alternanthera sessilis (L.) R.Br. ex DC. & Amaranthaceae & Ponnaganti kura & Herb & Leafy vegetable \\
\hline 21 & Alysicarpus monilifer (L.) DC & Fabaceae & & Decumbent herb & Weed \\
\hline 22 & Amaranthus dubius Mart. ex Thell. & Amaranthaceae & Thota kura & Herb & Leafy vegetable \\
\hline 23 & Amaranthus spinosus L. & Amaranthaceae & Mulla thotakura & Herb & Leafy vegetable \\
\hline 24 & Amorphophallus paeoniifolius (Dennst) Nicolson. & Araceae & Kanda gadda & Tuberous herb & Vegetable \\
\hline 25 & Andrographis echioides (L.) Nees & Acanthaceae & & Herb & Medicinal plant \\
\hline 26 & Andrographis paniculata (Burm.f.) Nees[ & Acanthaceae & Nela vemu & Herb & Medicinal plant \\
\hline 27 & Anisomeles malabarica (L.) R.Br. ex Sims & Lamiaceae & Malabar catmint/ Mogabiraku & Shrub & Medicinal plant \\
\hline 28 & Annona squamosa $\mathrm{L}$. & Annonaceae & Custard apple & Tree & Fruit yielding \\
\hline 29 & Antigonon leptopus Hook. \& Am. & Polygonaceae & Mexican creeper & Creeper & Ornamental plant \\
\hline 30 & Argemone mexicana $\mathrm{L}$. & Papaveraceae & Mexican poppy, Pichi kusuma & Herb & Medicinal plant \\
\hline 31 & Aristida adscensionis $\mathrm{L}$ & Poaceae & Chipurugaddi & Grass & Fodder \\
\hline 32 & Aristolochia indica $\mathrm{L}$. & Aristolochiaceae & Indian birth wort/Eshvara veru & Creeper & Medicinal plant \\
\hline 33 & Artocarpus heterophyllus Lam. & Moraceae & Jackfruit, Panasa & Tree & Fruit yielding \\
\hline 34 & Asparagus densiflorus (Kunth) Jessop & Asparagaceae & Asparagus & & Ornamental plant \\
\hline 35 & Asparagus racemosus Willd. & Asparagaceae & Satavari & Climber & Medicinal plant \\
\hline 36 & Asperagus setaceus (Kunth) Jessop & Asparagaceae & Asparagus fern & Climber & Ornamental plant \\
\hline 37 & Asplenium nidus $\mathrm{L}$. & Aspleniaceae & Nest fern & Herb & Ornamental plant \\
\hline
\end{tabular}




\begin{tabular}{|c|c|c|c|c|c|}
\hline 38 & Auricularia auricula-judae (Bull.) J.Schröt. & Auriculariaceae & Wood ear & Fungus & \\
\hline 39 & Azadirachta indica A.Juss. & Meliaceae & Vepa chettu, Neem & Tree & Medicinal plant, Wood yielding \\
\hline 40 & Azolla pinnata $\mathrm{R} . \mathrm{Br}$. & Salviniaceae & Moqueto fern, Water velvet & Herb & Biofertilizer \\
\hline 41 & Barleria cristata L. & Acanthaceae & Bluebell barleria & Shrub & Ornamental plant \\
\hline 42 & Bauhinia acuminata $\mathrm{L}$. & Fabaceae & White Orchid tree & Tree & Ornamental plant \\
\hline 43 & Bauhinia purpurea $\mathrm{L}$. & Fabaceae & Orchid tree, Butterfly tree & Tree & Ornamental plant \\
\hline 44 & Bauhinia variegata (L.)Benth. & Fabaceae & Mountain ebony & Tree & Ornamental plant \\
\hline 45 & Blumea lacera $\mathrm{L}$. & Asteraceae & & Herb & Medicinal plant \\
\hline 46 & Boerhavia diffusa L.nom.cons. & Nyctaginaceae & Punarnava & Decumbent herb & Medicinal plant \\
\hline 47 & Boerhavia erecta $\mathrm{L}$. & Nyctaginaceae & Erect spiderling & Herb & Weed \\
\hline 48 & Bombax ceiba $\mathrm{L}$. & Malvaceae & Cotton tree & Tree & Filling purpose \\
\hline 49 & Brachiaria remota (Retz.) Haines & Poaceae & & Grass & Fodder \\
\hline 50 & Brownea coccinea Jacq. & Fabaceae & Scarlet flame bean, rose of Venezuela & Tree & Medicinal plant \\
\hline 51 & Caesalpinia pulcherima (L.) Sw & Fabaceae & Peacock flower & Shrub & Ornamental plant \\
\hline 52 & Cajanus cajan (L.) Millsp. & Fabaceae & Redgram/Kandulu & & Pulses and vegetable \\
\hline 53 & Caladium bicolor (Aiton) Vent. & Araceae & Heart of Jesus & Tuberous herb & Ornamental plant \\
\hline 54 & Calotropis procera (Aiton) W.T.Aiton & Apocyanaceae & Jilledu, Crown flower & Shrub & Medicinal plant \\
\hline 55 & Calyptocarpus vialis Less. & Asteraceae & Creeping Cinderella weed & Herb & Weed \\
\hline 56 & Canna indica $\mathrm{L}$. & Cannaceae & Indian Shot & Tuberou herb & Ornamental plant \\
\hline 57 & Caralluma stalagmifera C.E.C.Fisch & Apocyanaceae & Dark purple caralluma & Succulent & Ornamental plant \\
\hline 58 & Caralluma umbellata / Boucerosia & Apocyanaceae & Kundeti kommulu & Succulent & Ornamental plant \\
\hline 59 & Cardiospermum halicacabum L. & Sapindaceae & Ballon vine & Climber & Medicinal plant \\
\hline 60 & Carica papaya $\mathrm{L}$. & Caricaceae & Papaya & Tree & Fruit yielding \\
\hline 61 & Carissa carandas $\mathrm{L}$. & Apocyanaceae & Bengal currant & Shrub & Fruit yielding \\
\hline 62 & Caryota urens $\mathrm{L}$. & Arecaceae & Fih tail palm & Tree & Alcoholic Beverage \\
\hline 63 & Catharanthus roseus G.Don & Apocyanaceae & Billaganneru & Herb & Medicinal plant \\
\hline 64 & Chamaecostus cuspidatus (Nees \& Mart)C.Specht \& D.W.Stev. & Costaceae & Inulin plant & Tuberous shrub & Medicinal plant \\
\hline 65 & \begin{tabular}{|c|} 
Cheilocostus speciosus (J.Konig) C.Specht \\
\end{tabular} & Costaceae & Crepe ginger & Tuberous shrub & Medicinal plant \\
\hline 66 & Chloris barbata $\mathrm{Sw}$. & Poaceae & & Grass & Fodder \\
\hline 67 & Chromolaena odorata (L.) R.M.King \&H.Rob. & Asteraceae & Kamourodda & Herb & Weed \\
\hline 68 & Cissus quadrangularis L. & Vitaceae & Devils back bone/ Nalleru & Succulent & Medicinal plant \\
\hline 69 & Cissus rotundifolia Vahl & Vitaceae & Arabian wax cissus & Succulent & Medicinal plant \\
\hline 70 & Citrus aurantium $\mathrm{L}$. & Rutaceae & Narinja & Tree & Fruit yielding \\
\hline 71 & Clematis gouriana Roxb.ex DC & Ranunculaceae & Indian Travellers Joy & Climber & Medicinal plant \\
\hline 72 & Cleome gynandra $\mathrm{L}$. & Cleomaceae & Vaminta & Herb & Medicinal plant \\
\hline 73 & Cleome rutidosperma DC. & Cleomaceae & Fringed spider flower & Herb & Weed \\
\hline 74 & Cleome viscosa $\mathrm{L}$. & Cleomaceae & Aian spider flower, tick weed & Herb & Medicinal plant \\
\hline 75 & Clitoria ternatea $\mathrm{L}$. & Fabaceae & Aian pigeonwings, sanku pushpam & Climber & Ornamental plant, Medicinal plant \\
\hline 76 & Coccinia grandis (L.) Voigt & Cucurbitaceae & Dondakaya & Climber & Vegetable \\
\hline 77 & Cocculus hirsutus (L.) Diels & Menispermaceae & Dusari teega, cheepuru teega & Climber & Medicinal plant \\
\hline 78 & Cocos nucifera $\mathrm{L}$. & Arecaceae & Coconut, Kobbari & Tree & Fruit yielding \\
\hline 79 & Codiaeum variegatum (L.) A.Juss. & Euphorbiaceae & Fire croton & Shrub & Ornamental plant \\
\hline 80 & Coleus amboinicus Lour. & Lamiaceae & Mexican mint & Succulent & Medicinal plant \\
\hline 81 & Combretum indicum (L.) DeFilipps & Combretaceae & Rangoon creeper & Climber & Ornamental plant \\
\hline 82 & Commelina attenuata K.D.Koenig ex Vahl & Commelinaceae & & Decumbent herb & Weed \\
\hline 83 & Commelina benghalensis $\mathrm{L}$. & Commelinaceae & Bengal dayflower & Decumbent herb & Weed \\
\hline 84 & Conocarpus erectus $\mathrm{L}$. & Combretaceae & Button wood & Tree & Ornamental plant \\
\hline 85 & Corchorus aestuansL. & Malvaceae & & Herb & Weed \\
\hline 86 & Cordyline fruticosa (L.) A.Chev. & Asparagaceae & ti plant, Palm lily & Shrub & $\begin{array}{l}\text { Food, traditional medicine } \\
\text { and Ornamental plant }\end{array}$ \\
\hline 87 & Cordia sebestena G.Forst. & Boraginaceae & Scarlet cordia, Geiger tree & Tree & Ornamental plant \\
\hline 88 & Cosmos sulphureus Cav. & Asteraceae & Sulfur cosmos & Herb & Dye yielding plant \\
\hline 89 & Costus woodsonii Maas. & Costaceae & Red button ginger & Tuberous shrub & Ornamental plant \\
\hline 90 & Crinum asiaticumL. & Amaryllidaceae & Poison bulb/ Chengalva puvvu & Tuberous shrub & Ornamental plant \\
\hline 91 & Crinum latifoliumL. & Amaryllidaceae & & Tuberou herb & Ornamental plant \\
\hline 92 & Crotalaria juncea $\mathrm{L}$. & Fabaceae & Janumu & Shrub & Fiber yielding \\
\hline 93 & Cryptostegia grandifloraRoxb.ex $\mathrm{R} \mathrm{Br}$. & Apocyanaceae & Rabbaru teega & Climber & Ornamental plant \\
\hline 94 & Curcuma amadaRoxb. & Zingiberaceae & Mamidi allamu & Tuberous herb & Medicinal plant \\
\hline 95 & Curcuma longa $\mathrm{L}$. & Zingiberaceae & Turmeric, Pasupu & Tuberous herb & Medicinal plant \\
\hline 96 & Cycas circinalis $\mathrm{L}$. & Cycadaceae & & Gymnosperm & Ornamental plant \\
\hline 97 & Cynodon dactylon(L.) Pers. & Poaceae & Garika & Grass & Fodder \\
\hline 98 & Cyperus rotundus $\mathrm{L}$. & Cyperaceae & Tunga & Tuberous herb & Weed \\
\hline 99 & Dactyloctenium aegyptium(L.) Willd. & Poaceae & Star grass & Grass & Fodder \\
\hline 100 & Desmodium gangeticum (L.) DC & Fabaceae & Salparni & Herb & Medicinal plant \\
\hline 101 & Desmodium triflorum (L.) DC & Fabaceae & Beggar weed & $\begin{array}{l}\text { Decumbent herb } \\
\text { Medicinal plant }\end{array}$ & \\
\hline 102 & Delonix regia (Hook.) Raf. & Fabaceae & Turayi & Tree & Ornamental plant \\
\hline 103 & Dicanthium annulatum (Forssk.) & Poaceae & & Grass & Fodder \\
\hline 104 & Digera muricata $($ L. $)$ Mart. & Amaranthaceae & & Decumbent herb & Medicinal plant \\
\hline 105 & Digitaria ciliaris (Retz.) Koel & Poaceae & & Grass & Fodder \\
\hline 106 & Digitaria sanguinalis (L.) & Poaceae & & Grass & Fodder \\
\hline 107 & Dioscorea esculenta (Lour.) Burkill & Dioscoreaceae & Yam & Creeper & vegetable \\
\hline 108 & $\begin{array}{l}\text { Dracaena angolensis (Welw.ex } \\
\text { Carriere) Byng \& Christenh }\end{array}$ & Asparagaceae & & Tuberous herb & Ornamental \\
\hline 109 & Dracaena fragrans (L.) Ker Gawl. & Asparagaceae & $\begin{array}{c}\text { Striped dracaena, } \\
\text { cornstalk dracaena }\end{array}$ & Shrub & Ornamental \\
\hline 110 & Dracaena reflexa Lam. & Asparagaceae & Song of India & Shrub & Ornamental \\
\hline 111 & Dracaena roxburghiana (Schult.\&Schult.f.) Byng \& Christenh. & Asparagaceae & & Tuberous herb & Medicinal plant \\
\hline 112 & Dracaena trifasciata (Prain) Mabb. & Asparagaceae & Mother in law tung, Snake plant & Tuberous herb & Ornamental \\
\hline 113 & Duranta erecta $\mathrm{L}$. & Verbenaceae & Golden duranta & Shrub & Ornamental \\
\hline 114 & Dypsis lutecens (H.Wendl.) Beentje \& J.Dransf & Arecaceae & Golden cane palm, Yellow Palm & Tree & Ornamental \\
\hline 115 & Eclipta alba Hassk. & Asteraceae & Guntagalagaraaku & Herb & Medicinal plant \\
\hline 116 & Elaeis guineensis Jacq. & Arecaceae & Palm oil & Tree & Oil yielding plant \\
\hline 117 & Elaeocarpus ganitrus Roxb.ex G.Don & Elaeocarpaceae & Rudraksha & Tree & \\
\hline 118 & Eleusine indica (L.) Gaertn. & Poaceae & & Grass & Fodder \\
\hline 119 & Ergostris ciliaris (L.) & Poaceae & & Grass & Fodder \\
\hline 120 & Euphorbia geneculeta Ortega & Euphorbiaceae & & Herb & Weed \\
\hline 121 & Euphorbia hirtaL. & Euphorbiaceae & Garden spurge & Herb & Medicinal plant \\
\hline
\end{tabular}




\begin{tabular}{|c|c|c|c|c|c|}
\hline 122 & Euphorbia milii Des Moul. & Euphorbiaceae & & Succulent & Ornamental \\
\hline 123 & Euphorbia splendens Bojer ex Hook. & Euphorbiaceae & & Succulent & Ornamental \\
\hline 124 & Euphorbia tirucalli L. & Euphorbiaceae & Pencil tree & Tree & Medicinal plant \\
\hline 204 & Euphorbia tithymaloides L. & Euphorbiaceae & & Succulent & Ornamental plant \\
\hline 125 & Euphorbia trigona Roxb. & Euphorbiaceae & & Succulent & Ornamental, Medicinal plant \\
\hline 126 & Evolulus nummularius (L.) L. & Convolulaceae & & Decumbent herb & Medicinal plant \\
\hline 127 & Excoecaria bicolor (Hassk.) Zoll.ex.Hassk. & Euphorbiaceae & & Shrub & Ornamental \\
\hline 128 & Ficus carica $\mathrm{L}$. & Moraceae & Common fig/ Anjeer & Tree & Fruit yielding \\
\hline 129 & Ficus hispida Blanco & Moraceae & Opposite leaf fig/ Bomma medi & Tree & \\
\hline 130 & Ficus racemosaWilld. & Moraceae & Cluster fig/ Medi chettu & Tree & Medicinal plant \\
\hline 131 & Ficus religiosa Forssk. & Moraceae & Raavi/ Peepal tree & Tree & Medicinal plant \\
\hline 132 & Foeniculum vulgare Mill. & Apiaceae & Fennel/Sompu & Herb & Medicinal plant \\
\hline 133 & Licula grandis H.Wendl. & Arecaceae & Fan Palm & Tree & Ornamental \\
\hline 134 & Furcraea foetida (L.) Haw. & Asparagaceae & Mauritius-hemo & Succulent & Fiber yielding \\
\hline 135 & Galphimia glauca Bartl. & Malphigiaceae & & Shrub & Ornamental \\
\hline 136 & Ganoderma lucidum (Curtis) P.Krast.(1881) & Ganodermataceae & Bracket fungi/ Shelf mushroom & Fungus & \\
\hline 137 & Gomphrena celosioides Mart. & Amaranthaceae & & Decumbent herb & Weed \\
\hline 138 & Gomphrena globosa L. & Amaranthaceae & Bogada banti/Globe amaranth & Herb & Ornamental \\
\hline 139 & Graptophyllam pictum $(\mathrm{L}$.$) Griff.$ & Acanthaceae & Caricature plant & Shrub & Ornamental \\
\hline 140 & Hedychium coronarium J.Koenig & Zingiberaceae & White ginger lily/Butterfly lily & Tuberous shrub & Medicinal plant \\
\hline 141 & Hamelia patens Jacq. & Rubiaceae & Fire bush & Tree & Ornamental \\
\hline 142 & Heteropogon contortus (L.) P. Beauv.ex.Roem. \& Schult. & Poaceae & Blacks spear grass & Grass & Fodder \\
\hline 143 & Hibicus lobatus (Murray) Kuntze & Malvaceae & Ataka naara & Herb & Weed \\
\hline 144 & Hibiscus tiliaceus $\mathrm{L}$. & Malvaceae & Beach Hibiscus & Tree & Ornamental \\
\hline 145 & Hippeastrum correiense (Bury) Worsley & Amaryllidaceae & Easter lily & Tuberous herb & Ornamental \\
\hline 146 & Holoptelea integrifolia (Roxb.) Planch. & Ulmaceae & Nemalinara chettu/ nali & Tree & Wood yielding \\
\hline 147 & Hydrilla verticillata (L.f.) Royle & Hydrocharitaceae & Waterthyme & Aquatic plant & Medicinal plant \\
\hline 148 & Hymenocallis latifolia (Mill.) Planch. & Amaryllidaceae & Spider lily & Tuberous herb & Ornamental plant \\
\hline 149 & Indigofera linifolia (L.f.) Retz. & Fabaceae & Narrow leaf indigo & Decumbent herb & Weed \\
\hline 150 & Indigofera linnaei Ali & Fabaceae & Birdsville indigo(L.f.) Retz & Decumbent herb & Weed \\
\hline 151 & Ipomoea aquatica Forssk. & Convolulaceae & Neeti thuti & Aquatic plant & Leafy vegetable \\
\hline 152 & Ipomoea hederifolia $\mathrm{L}$. & Convolulaceae & Scarlet morning glory, & Climber & Ornamental \\
\hline 153 & Ipomoea pes-tigridis $\mathrm{L}$. & Convolulaceae & Tigers paw & Creeper & Weed \\
\hline 154 & Ixora coccinea $\mathrm{L}$. & Rubiaceae & Nuruvarahalu & Shrub & Ornamental \\
\hline 155 & Jasminum sambac (L.) Ait. & Oleaceae & Jasmine/ Malli & Shrub & Ornamental \\
\hline 156 & Jatropha gossypifolia L. & Euphorbiaceae & Nepalam & Shrub & Medicinal plant \\
\hline 157 & Jatropha podagrica Hook. & Euphorbiaceae & Coral plant, Buddha belly plant & Succulent & Ornamental \\
\hline 158 & Kaempferia galanga $\mathrm{L}$. & Zingiberaceae & kachoralu & Tuberous shrub & Medicinal plant \\
\hline 159 & Kaempferia rocoeana Wall. & Zingiberaceae & Peacock ginger & Tuberous herb & Ornamental plant \\
\hline 160 & Kaempferia rotunda $\mathrm{L}$. & Zingiberaceae & Bhumi champa & Tuberous herb & Medicinal plant \\
\hline 161 & Kleinia grandiflora (Wallich ex DC) N.Rani. & Asteraceae & Large flower Kleinia & Succulent & Ornamental plant \\
\hline 162 & Kalanchoe pinnata (Lam.)Pers. & Crassulaceae & Miracle leaf/ Ranapalaku & Succulent & Medicinal plant \\
\hline 163 & Kleinhovia hospita L. & Malvaceae & Guest tree & Tree & Medicinal plant \\
\hline 164 & Lagerstroemia speciosa (L.)Pers. & Lythraceae & Pride of India & Shrub & Ornamental plant \\
\hline 165 & Lantana camara $\mathrm{L}$. & Verbenaceae & Gaju poolu & Herb & Ornamental plant \\
\hline 166 & Lawsonia inermis $\mathrm{L}$. & Lythraceae & Henna tree, Gorintaaku & shrub & Dye yielding plant \\
\hline 167 & Lediborea revoluta (L.f) Jessop & Asparagaceae & South Indian Skill. & Tuberous herb & Medicinal plant \\
\hline 168 & Leucaena leucocephala (Lam.) de wit & Fabaceae & White lead tree/ Kanti Subabul & Tree & \\
\hline 169 & Licula orbicularis Becc. & Arecaceae & & Palm & Ornamental plant \\
\hline 170 & Livistona chinensis L. & Arecaceae & Chinese Fan palm & Palm & Ornamental plant \\
\hline 171 & Ludwigia adscendens (L.) H.Hara & Onagraceae & the evening primerose & Aquatic plant & Vegetable \\
\hline 172 & Manihot esculenta Crantz. & Euphorbiaceae & Karrapendalamu & Tuberous shrub & vegetable \\
\hline 173 & Manilkara hexandra (Roxb.) Dubard. & Sapotaceae & Pala chettu & Tree & Wood yielding \\
\hline 174 & Mariscus squarrosus (L.) C.B.Clarke. & Cyperaceae & Scarlet morning glory, & Herb & Weed \\
\hline 175 & Mentha arvensis $\mathrm{L}$. & Lamiaceae & Pudeena & Decumbent herb & Medicinal plant \\
\hline 176 & Microstachys chamaelea (L.) Mull.Arg. & Euphorbiaceae & & Herb & Weed \\
\hline 177 & Millingtonia hortensis L.f. & Bignoniaceae & Punnaga & Tree & Ornamental \\
\hline 178 & Mimuops elengi $\mathrm{L}$. & Sapotaceae & Pagada/ Spanish cherry & Tree & Fruit yielding \\
\hline 179 & Mirabilis jalapa $\mathrm{L}$. & Nyctaginaceae & Chandra kanta/4 o clock plant & Tuberous herb & Medicinal plant \\
\hline 180 & Mollugo nudicaulis Lam. & Aizoaceae & Naked-stem carpetweed & Herb & Medicinal plant \\
\hline 181 & Moringa oleifera Lam. & Moringaceae & Mulaga/ Drumstick plant & Tree & vegetable \\
\hline 182 & Muntingia calabura $\mathrm{L}$. & Muntingiaceae & Strwberry tree & Tree & Medicinal plant \\
\hline 183 & Murraya koenigii (L.) Sprengel & Rutaceae & Cury leaf tree & Tree & Medicinal plant \\
\hline 184 & Murraya paniculata (L.) Jack. & Rutaceae & Orange Jasmine & Tree & Ornamental \\
\hline 185 & Musa paradiiaca $\mathrm{L}$. & Musaceae & Plantain tree/Banana & Tree & Fruit yielding \\
\hline 186 & Mussaenda erythrophylla Schumach.\& Thonn & Rubiaceae & Red flag bush & Shrub & Ornamental \\
\hline 187 & Najas indica (Willd.) Cham & Hydrocharitaceae & Najas grass & Aquatic plant & Aquarium plant \\
\hline 188 & Nerium indicum Mill. & Apocyanaceae & Kaliveru & Shrub & Ornamental \\
\hline 189 & Nerium oleander $\mathrm{L}$. & Apocyanaceae & Oleander & Shrub & Ornamental \\
\hline 190 & Nyctanthes arbor-tritis $\mathrm{L}$. & Oleaceae & Coral jasmine/ Parijaatam & Tree & Medicinal plant \\
\hline 191 & Nymphaea nouchali Burm.f. & Nymphaeaceae & Blue lotus & Aquatic Plant & Medicinal plant \\
\hline 192 & Ocimum americanum $\mathrm{L}$. & Lamiaceae & American basil & Aromatic herb & Medicinal plant \\
\hline 193 & Ocimum basilicum $\mathrm{L}$. & Lamiaceae & Sweet basil & Aromatic herb & Medicinal plant \\
\hline 194 & Ocimum tenuiflorum $\mathrm{L}$. & Lamiaceae & Holy basil & Aromatic herb & Medicinal plant \\
\hline 195 & Oldenlandia herbacea DC. & Rubiaceae & & Herb & Weed \\
\hline 196 & Oldenlandia umbellata Steud. & Rubiaceae & & Herb & Weed \\
\hline 197 & Oreodox regia Boiss. & Arecaceae & & Palm & Ornamental plant \\
\hline 198 & Oxalis corniculata $\mathrm{L}$. & Oxalidaceae & Procumbent yellow sorrel, pulichinta & Decumbent herb & Medicinal plant \\
\hline 199 & Oxalis latifolia Kunth. & Oxalidaceae & garden pink sorrel & Tuberous herb & Weed \\
\hline 200 & Oxalis triangularis A.St.Hil. & Oxalidaceae & False shamrock & Tuberous herb & Ornamental plant \\
\hline 201 & Pancratium longiflorum Roxb.ex Ker Gawl. & Amaryllidaceae & & Tuberous herb & Ornamental plant \\
\hline 202 & Parthenium hysterophorus Adans. & Asteraceae & & Shrub & Weed \\
\hline 203 & Passiflora foetida $\mathrm{L}$. & Passifloraceae & & Climber & Medicinal plant \\
\hline 205 & Peltophorum pterocarpum (DC.) K.Heyne & Fabaceae & Pacha Turayi, Yellow flame tree & Tree & Ornamental plant \\
\hline 206 & Pennisetum setaceum (Forssk.) Chiov & Poaceae & crimson fountaingrass & Grass & Ornamental plant \\
\hline 207 & Pereskia grandifolia subsp. grandifolia & Cactaceae & & Shrub & Ornamental plant \\
\hline 208 & Pergularea daemia subsp. Garipensis (E.Mey.) Goyder & Apocyanaceae & Dushtapu teega & Climber & Medicinal plant \\
\hline 209 & Phyllanthus acidus (L.) Skeels & Phyllanthaceae & Country gooseberry & Tree & Fruit yielding \\
\hline
\end{tabular}




\begin{tabular}{|c|c|c|c|c|c|}
\hline 210 & Phyllanthus emblica $\mathrm{L}$. & Phyllanthaceae & Indian gooseberry, amla & Tree & Fruit yielding \& Medicinal plant \\
\hline 211 & Phyllanthus madras pattens & Phyllanthaceae & & Herb & Medicinal plant \\
\hline 212 & Phyllanthus niruri $\mathrm{L}$. & Phyllanthaceae & Nelausiri & Herb & Medicinal plant \\
\hline 213 & Phyllanthus reticulatusPoir. & Phyllanthaceae & Purudu kayalu & Shrub & Medicinal plant \\
\hline 214 & Philodendron erubescens K.Koch \& Augustin & Araceae & blushing philodendron & Creeper & Ornamental plant \\
\hline 215 & Physalis minima $\mathrm{L}$. & Solanaceae & Budda budasara & Herb & Medicinal plant \\
\hline 216 & Pistia stratiotes $\mathrm{L}$. & Araceae & Water cabbage & Aquatic plant & Ornamental plant \\
\hline 217 & Pithecellobium dulce (Roxb.) Benth. & Fabaceae & Seema chintha & Tree & Fruit yielding \\
\hline 218 & Plumeria pudica Jacq. & Apocyanaceae & Temple tree & Tree & Ornamental plant \\
\hline 219 & Plumeria rubraL. & Apocyanaceae & & Tree & Ornamental plant \\
\hline 220 & Polianthes tuberosa L. & Asparagaceae & Tuberose, lily & \begin{tabular}{|l|} 
Tuberous herb \\
\end{tabular} & Ornamental plant \\
\hline 221 & Polyalthia longifolia (Sonn.) Thwaites & Annonaceae & Naramamidi & Tree & Ornamental plant \\
\hline 222 & Pongamia pinnata (L.) Pierre. & Fabaceae & Ganuga, Kamu chettu & Tree & Medicinal plant \\
\hline 223 & Pteris L. & Pteridaceae & & Herb & Ornamental plant \\
\hline 224 & Punica granatum $\mathrm{L}$. & Punicaceae & Daanimma, Pomegranate & Tree & Fruit yielding \\
\hline 225 & Ravenia spectabilis (Lindl.) Engl. & Rutaceae & & Shrub & Ornamental plant \\
\hline 226 & Rhodophiala rosea (Sweet) Traub & Amaryllidaceae & Easter lily & Tuberous herb & Ornamental plant \\
\hline 227 & Rhynchosia minima $(\mathrm{L}$.$) DC$ & Fabaceae & Least snout-bean, burn-mouth-vine & Climber & Weed \\
\hline 228 & Rosa indica $\mathrm{L}$. & Rosaceae & Gulabi, Rose & Shrub & Ornamental plant \\
\hline 229 & Rottboellia exaltata L.f. & Poaceae & Itch grass & Grass & Fodder \\
\hline 230 & Ruellia tuberosa $\mathrm{L}$. & Acanthaceae & Fever root, Snapdragon root & Tuberous herb & Weed \\
\hline 231 & Salvinia minima Baker. & Salviniaceae & water spangles & Aquatic fern & Bioagent for wastewater treatment, feed \\
\hline 232 & Sapindus marginatus Willd. & Sapindaceae & Kunkullu, Soapnut & Tree & Medicinal plant \\
\hline 233 & Scadoxus multiflorus (Martyn) Raf. & Amaryllidaceae & Blood lily & Tuberous herb & Ornamental plant \\
\hline 234 & Scoparia dulcis $\mathrm{L}$. & Scropulariaceae & Dakshini, Sweet broom & Herb & Medicinal plant \\
\hline 235 & Senna alata (L.) Roxb. & Fabaceae & Candle bush, ringworm shrub & Shrub & Medicinal plant \\
\hline 236 & Senna fistula $\mathrm{L}$. & Fabaceae & Indian laburnum, Rela & Tree & Medicinal plant \\
\hline 237 & Senna siamea (Lam.) Irwin et Barneby. & Fabaceae & & Tree & Ornamental \\
\hline 238 & Setaria verticillata (L.) P.Beauv. & Poaceae & Bristly foxtail & Grass & Fodder \\
\hline 239 & Psidium guajava L. & Myrtaceae & Guava, Jamakaya & Tree & Fruit yielding \\
\hline 240 & Solanum nigrum L. & Solanaceae & Kamanchi & Herb & Medicinal plant \\
\hline 241 & Spathodea campanulata P.Beauv. & Bignoniaceae & Tulip tree & Tree & Wood yielding \\
\hline 242 & Spermacoce hispida $\mathrm{L}$ & Rubiaceae & Shaggy button weed & Herb & Medicinal plant \\
\hline 243 & Spirodela polyrhiza (L.) Schleid. & Araceae & Common duckmeat & Aquatic Plant & Animal feed, biofuel, bioremediation \\
\hline 244 & Swietenia mahagoni (L.) Jacq. & Meliaceae & West Indian mahogany & Tree & Wood yielding \\
\hline 245 & Syzygium cumini (L.) Skeels. & Myrtaceae & Neredu & Tree & Fruit yielding \\
\hline 246 & Syzygium malaccense (L.) Merr. \& L.M.Perry. & Myrtaceae & Malay apple & Tree & Fruit yielding \\
\hline 247 & Tagetus erecta $\mathrm{L}$. & Asteraceae & Banthi & shrub & Ornamental \\
\hline 248 & Talinum fruticosum (L.) Juss. & Talinaceae & Englih bachali & Succulent & Leafy vegetable \\
\hline 249 & Tamarindus indica $\mathrm{L}$. & Fabaceae & Tamarind, Chintha & Tree & vegetable \\
\hline 250 & Tecoma stans (L.) Juss. ex Kunth & Bignoniaceae & Yellow bells & Tree & Wood yielding \\
\hline 251 & Terminalia arjuna (Roxb.) Wight \& Arn. & Combretaceae & Arjun tree, tella maddi & Tree & Wood yielding \\
\hline 252 & Terminalia catappa $\mathrm{L}$. & Combretaceae & Indian Almond, Badam & Tree & Nut yielding \\
\hline 253 & Terminalia chebula Retz. & Combretaceae & Myrobalan, Karakkaya & Tree & Medicinal plant \\
\hline 254 & Tinospora cordifolia (Thunb.) Miers & Minispermaceae & Thippatheega & Climber & Medicinal plant \\
\hline 255 & Tradescantia spathacea $\mathrm{Sw}$ & Commelinaceae & Boat lily, Moses in the Cradle & Herb & Ornamental \\
\hline 256 & Tradescantia pallida (Rose) D.R.Hunt & Commelinaceae & Purple heart & Decumbent herb & Ornamental \\
\hline 257 & Tradescantia zebrena (Schinz) D. R. Hunt & Commelinaceae & inch plant or wandering Jew & Decumbent herb & Ornamental \\
\hline 258 & Trianthema decandra $\mathrm{L}$. & Aizoaceae & Gangabayala kura & Decumbent herb & Leafy vegetable \\
\hline 259 & Trianthema triquetra Rottler \& Willd (Tt.Cr) & Aizoaceae & Galijeru & Decumbent herb & Leafy vegetable \\
\hline 260 & Tribulus terestris $\mathrm{L}$. & Zygophyllaceae & Palleru & Decumbent herb & Medicinal plant \\
\hline 261 & Trichodesma zeylanicum (Burm.f.) R.Br. & Boraginaceae & Pedda gurragutti, Camel bush & Herb & Medicinal plant \\
\hline 262 & Tridax procumbens $\mathrm{L}$. & Asteraceae & Gaddichemanthi & Herb & Medicinal plant \\
\hline 263 & Typhonium inopinatum Prain. & Araceae & & Tuberous herb & Medicinal plant \\
\hline 264 & Typhonium roxburghii Schott. & Araceae & Dwarf voodoo lily & Tuberous herb & Medicinal plant \\
\hline 265 & Typhonium trilobatum (L.) Schott. & Araceae & Bengal Arum & Tuberous herb & Medicinal plant \\
\hline 266 & Urochloa panicoides P.Beauv. & Poaceae & & Grass & Fodder \\
\hline 267 & Vernonia cenerea $($ L.) Less & Asteraceae & Sahadevi & Herb & Medicinal plant \\
\hline 268 & Vitex negundo L. & Lamiaceae & Vavili & Tree & Medicinal plant \\
\hline 269 & Washingtonia filifera (Linl.) H.Wendl. & Arecaceae & & Palm & Ornamental \\
\hline 270 & Withania somnifera $(\mathrm{L}$.$) Dunal$ & Solanaceae & Ashwagandha & shrub & Medicinal plant \\
\hline 271 & Yucca gloriosa $\mathrm{L}$ & Asparagaceae & & Shrub & Ornamental \\
\hline 272 & Zephyranthes candida (Lindl.) Herb. & Amaryllidaceae & Rain lily/Zephyr lily & Tuberous herb & Ornamental \&Medicinal plant \\
\hline 273 & Zephyranthes carinata Herb. & Amaryllidaceae & Rain lily/Zephyr lily & Tuberous herb & Ornamental \&Medicinal plant \\
\hline 274 & Zephyranthes citrina Baker & Amaryllidaceae & Rain lily/Zephyr lily & Tuberous herb & Ornamental \&Medicinal plant \\
\hline 275 & Zephyranthes rosea Lindl. & Amaryllidaceae & Rain lily/Zephyr lily & Tuberous herb & Ornamental \&Medicinal plant \\
\hline 276 & Zingiber cassumunar Roxb. & Zingiberaceae & Karu allamu & Tuberous herb & Medicinal plant \\
\hline 277 & Zingiber montanum (J.König) Link ex A.Dietr. & Zingiberaceae & Adavi allamu & Tuberous herb & Medicinal plant \\
\hline 278 & Zingiber officinale Roscoe. & Zingiberaceae & Allamu, Ginger & Tuberous herb & Medicinal plant \\
\hline 279 & Ziziphus jujuba Mill. & Rhamnaceae & Regu & Tree & Fruit yielding \\
\hline
\end{tabular}

Table 2. Different plant groups in study area with number of families and species

\begin{tabular}{|c|c|c|c|}
\hline S. No & Category & Number of families & Number of Species \\
\hline 1 & Fungi & 2 & 2 \\
\hline 2 & Pteridophytes & 3 & 5 \\
\hline 3 & Gymnosperm & 1 & 1 \\
\hline 4 & Dicots & 53 & 184 \\
\hline 5 & Monocots & 12 & 89 \\
\hline
\end{tabular}




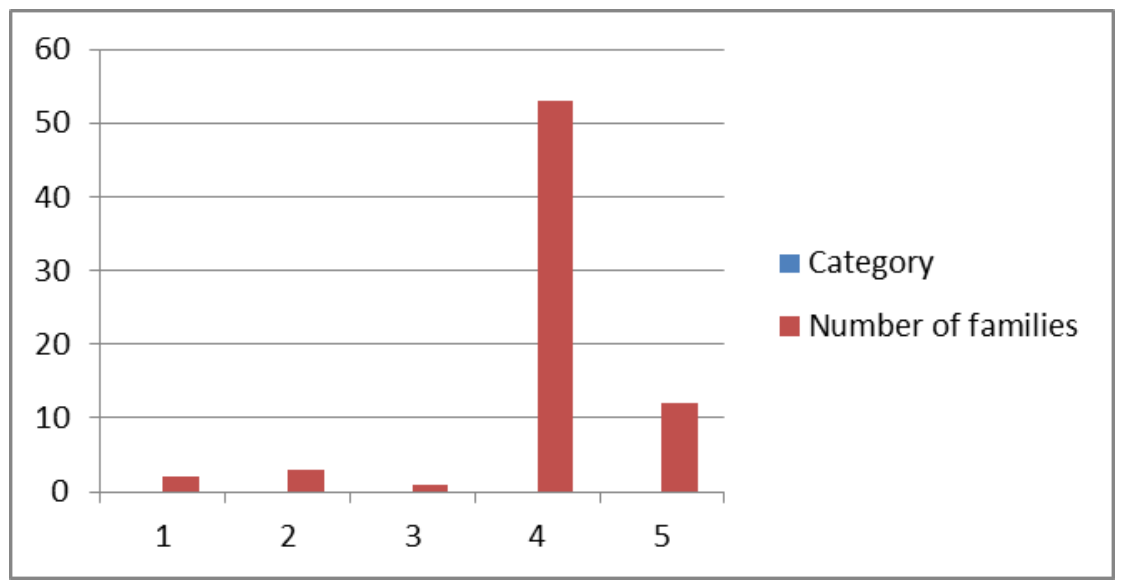

Fig 1. Graph showing dominant plant groups based on number of families

Table 3: 10 dominant families

\begin{tabular}{|c|c|c|}
\hline S. No & Name of the Family & Number of Species \\
\hline 1 & Fabaceae & 24 \\
\hline 2 & Euphorbiaceae & 16 \\
\hline 3 & Apocyanaceae & 15 \\
\hline 4 & Poaceae & 15 \\
\hline 5 & Asparagaceae & 14 \\
\hline 6 & Asteraceae & 11 \\
\hline 7 & Zingiberaceae & 11 \\
\hline 8 & Amaryllidaceae & 11 \\
\hline 9 & Araceae & 10 \\
\hline 10 & Arecaceae & 9 \\
\hline
\end{tabular}

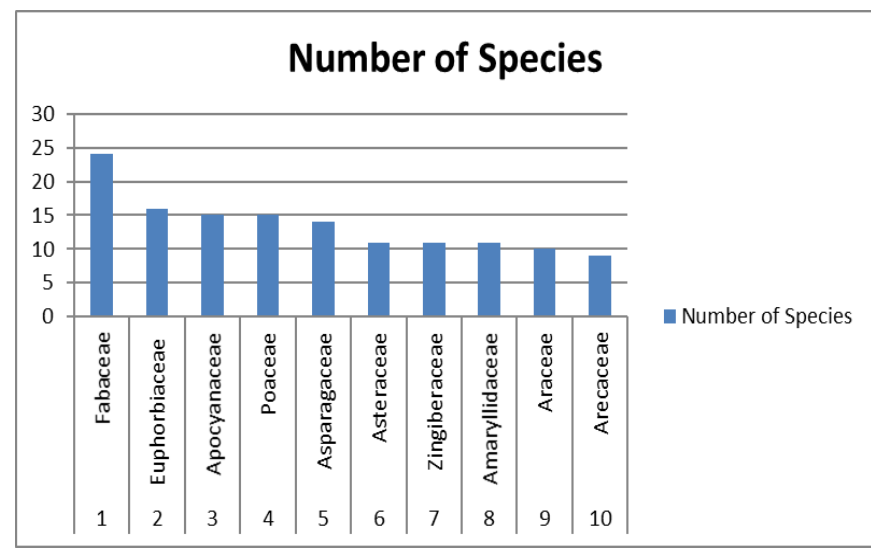

Fig 2. Species richness in 10 dominant families

Table 4. Major life forms in Fort Campus

\begin{tabular}{|c|c|}
\hline Herbs & $48.50 \%$ \\
\hline Trees & $30.70 \%$ \\
\hline Shrubs & $14 \%$ \\
\hline Climbers & $7.80 \%$ \\
\hline Hydrophytes & $2.50 \%$ \\
\hline
\end{tabular}

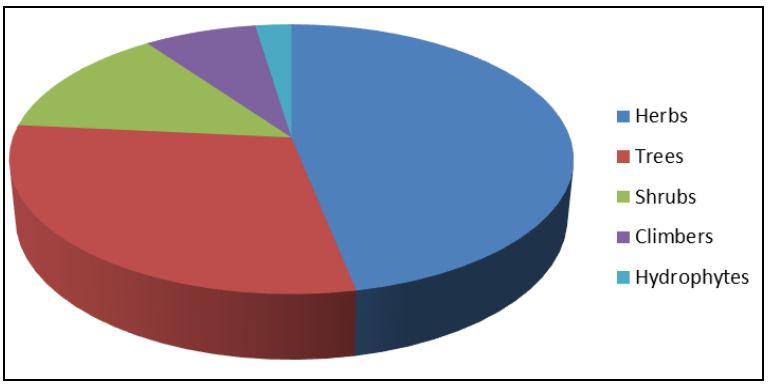

Fig 3: Graph showing Major life forms in Fort Campus
Table 5: Economically important plants

\begin{tabular}{|c|c|}
\hline Medicinal plants & $\mathbf{3 3 \%}$ \\
\hline Ornamental plants & $21 \%$ \\
\hline Weeds & $9 \%$ \\
\hline Fruit yielding plants & $6.40 \%$ \\
\hline Vegetable plants & $5 \%$ \\
\hline Fodder plants & $5 \%$ \\
\hline Wood yielding plants & $2.80 \%$ \\
\hline Fibre yielding plants & $1 \%$ \\
\hline Dye yielding plants & $0.70 \%$ \\
\hline Miscellaneous plants & $14 \%$ \\
\hline
\end{tabular}

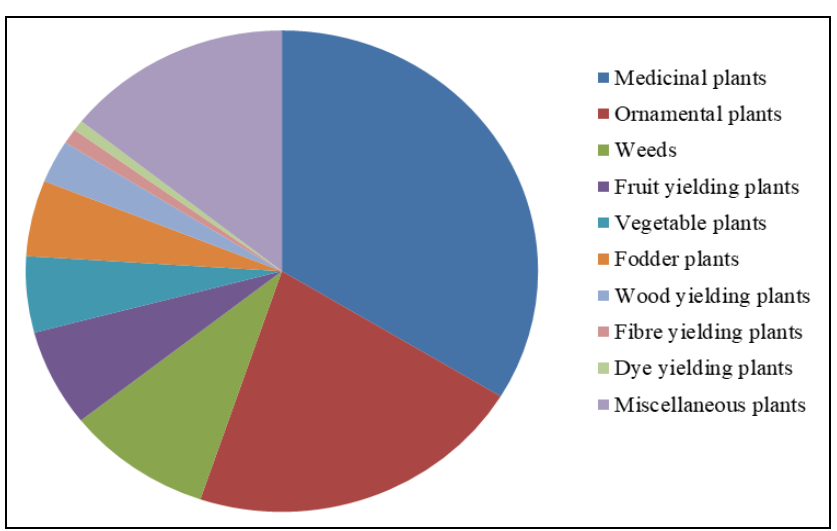

Fig 5. Graph showing economically important plants

\section{Acknowledgements}

Author has grateful to late Sri Dr. P.V. G. Raju garu, and also special thanks to Dr. J. Prakasa Rao, Andhra University, Visakhapatnam, for he has seeded a thought for this paper and the seed came to fruition.

\section{Conclusion}

As mentioned above, fort campus is suitable for growing all kinds of plants, which are native plants, invasive plants, and introduced plants (rare and endangered). For ex-situ conservation fort campus is the best place, here there is no anthropological and animal polluted activities. Especially the Buruju (Tower) area is more suitable for growing rare and endangered plants for conservation. Author has strongly recommended that if there is any projects on Biodiversity conservation and plant conservation of rare and endangered plant species choose this area for conservation.

\section{References}

1. Prakasarao J. Plant diversity and their significance of 
Adikavi Nannaya University Campus Asian Journal of Plant Science and Research. 2016;6(3):43-54.

2. Prameela R. Uncultivated fodder grass for cattle Tropical Plant Research. 2016;3(3):533-535.

3. Prameela R, Swamy J, Venkaiah M. Typhonium inopinatum Prain (Araceae): An addition to the flora of Andhra Pradesh Annals of plant Sciences 2018;7(4):2147-2149.

4. Prameela R, Venkaiah M, Swamy J, Prakasarao J. Amaryllids of Andhra Pradesh, India Tropical Plant Research. 2020;7(3):594-603.

5. Pullaiah T. Flora of Andhra Pradesh (India), Scientific Publishers, Jodhpur. 2018, 5.

6. Rao JP, Aniel Kumar O, Venkaiah M. Diversity and Distribution of Tree species in Andhra University Campus, Visakhapatnam City, Andhra Pradesh, India. National Seminar on Genomes, Environment \& Human Welfare-Retrospect and Prospect. Department of Botany, Andhra University, Visakhapatnam. 2013.

7. Reddy CS, Bhagyanarayana G, Reddy KN, Raju VS. Invasive Alien Flora of India Biodiversity News of Andhra Pradesh. 2008;(2):2-3.

8. Rolla Rao S, Sriramulu HS. Flora of Srikakulam district, Andhra Pradesh, India (Flora of India Series). Indian Botanical Society, Meerut. 1986.

9. Singh Aarvind. International Journal of Bioinformatics and Biomedical Engineering. 2015;1(3):222-236.

10. Venkaiah M. Studies on the Vegetation and Flora of Vizianagaram district, Andhra Pradesh, Andhra University, Visakhapatnam. 2004. 